\title{
Cartographie de dose gamma en champ mixte et en milieu modérateur par thermoluminescence impulsionnelle
}

\author{
H. SERVIÈRE*, F. VETTÈSE**, J. FESQUET***, J. GASIOT***
}

(Manuscrit reçu le 6 février 1996, révisé le 5 décembre 1996, accepté le 18 décembre 1996)

RÉSUMÉ Ce travail présente une application de dosimétrie cartographique gamma à l'intérieur d'un mannequin anthropomorphe irradié en champ mixte neutrongamma (avec $\gamma / \boldsymbol{n}=\mathbf{0 , 1 6}$ dans l'air). Les cartes de dose gamma sont obtenues par chauffage laser impulsionnel d'une plaque d'alumine thermoluminescente (TL), dont les propriétés sont étudiées. L'alumine, du fait de sa faible sensibilité aux neutrons thermiques, est bien adaptée à la mesure de la dose gamma dans un milieu où l'abondance des neutrons thermiques peut atteindre $80 \%$ de la fluence neutronique totale sous $5 \mathrm{~cm}$ de modérateur. La répartition de la dose gamma dans le mannequin est comparée à celle que l'on obtient dans un champ à composante gamma prépondérante $(\gamma / n=11)$.

ABSTRACT Gamma dose mapping in mixte neutron-gamma field and moderator medium by laser heating thermoluminescence.

The present work shows an application of gamma dose mapping inside an anthropomorphous phantom, irradiated in a mixed neutron-gamma radiation field $(\gamma / n=0.16$ in air). The gamma dose mappings are obtained with point by point laser heated readouts of a thermoluminescent alumina plate. Because of low thermal neutron sensitivity, aluminium oxide is a convenient material for gamma dose measurements in an environment where the quantity of thermal neutrons can reach $80 \%$ of the total neutron fluence under a $5 \mathrm{~cm}$ thick moderator. The gamma dose distribution in the phantom is compared to the distribution measured in a dominating gamma component radiation field $(\gamma / n=11$ in air $)$.

\section{Introduction}

C'est à partir des années cinquante, à la suite des travaux donnant les bases théoriques de la luminescence stimulée thermiquement (Randall et Wilkins, 1945), que les études sur la thermoluminescence (TL) appliquée à la dosimétrie des rayonnements ionisants ont pris leur essor. Ce développement s'est accru considérablement dans le milieu des années soixante (Cameron et al., 1968) avec la production en grande quantité de matériaux thermoluminescents adaptés aux applications dosimétriques (LiF, $\mathrm{CaF}_{2}:$ Dy...), ainsi que par la

\footnotetext{
* Institut de protection et de sâreté nucléaire, Département de protection de la santé de l'Homme et de dosimétrie, Service de dosimétrie, BP. 6, 92265 Fontenay-aux-Roses cedex.

** D.G.A./D.R.E.T., Centre d'études du Bouchet/D.P.N., BP. 3, 91710 Vert-le-Petit cedex.

*** Université de Montpellier II, Centre d'électronique de Montpellier II (UMR 5507), Place Eugène Bataillon, 34095 Montpellier cedex 05.
} 
mise au point de lecteurs utilisant l'électronique pour les techniques de chauffage et de détection. La dosimétrie par thermoluminescence présente de nombreux avantages parmi lesquels un seuil de détection bas (10 micrograys pour le sulfate de calcium), une dynamique importante (linéarité sur 7 décades) et une mise en cuvre aisée.

Une étape nouvelle fut franchie lorsque Bräunlich et al. (1981) utilisèrent pour la première fois un faisceau laser infrarouge (IR) comme moyen de chauffage. Ce dernier a permis l'accroissement d'un facteur mille de la vitesse de chauffage et corrélativement de l'intensité du signal de TL. Le temps de lecture et le volume de matériau TL à chauffer peuvent, à performance dosimétrique équivalente, être réduit dans la même proportion. Ces deux propriétés ont permis la mise au point d'un système de cartographie de dose bêta et gamma (Gasiot et al., 1982). Cette technique mise au point à Montpellier, employée avec l'alumine TL comme capteur dosimétrique, permet d'élargir le champ d'application de la cartographie de dose gamma en mannequin dans un champ mixte neutron-gamma. En effet, dans un tel champ de rayonnement, les matériaux thermoluminescents les plus courants conduisent à des erreurs importantes en raison de leur sensibilité aux neutrons.

Ce procédé de cartographie a été développé à la demande de radiobiologistes, qui souhaitaient connaître les répartitions respectives des doses gamma et neutron à l'intérieur d'un sujet irradié en champ mixte afin de relier le syndrome de l'irradiation aux dommages produits au niveau des divers territoires organiques.

L'information acquise grâce à la cartographie de dose par chauffage laser est continue et non pas ponctuelle comme c'est le cas des mesures faites avec des dosimètres discrets. L'utilisateur a ainsi accès non seulement à une mesure précise de la dose dans les tissus même s'ils sont de densité différente, mais aussi à sa répartition sur une surface de plusieurs centaines de centimètres carrés.

Même exposé à un rayonnement purement neutronique (ce qui, au demeurant, constitue une situation exceptionnelle), les organes internes sont soumis à un champ mixte du fait des diffusions inélastiques et des captures radiatives qui génèrent un rayonnement gamma secondaire. La contribution de ces derniers à la dose totale peut devenir prépondérante au niveau des organes profonds dans le cas de champ de fission.

Après avoir montré pourquoi l'alumine thermoluminescente permet de mesurer la dose gamma en champ mixte, nous décrivons les propriétés dosimétriques de l'alumine en poudre utilisée pour la réalisation de nos films TLD destinés à la cartographie. Nous comparerons ensuite les cartographies de dose obtenues à l'intérieur d'un mannequin anthropomorphe irradié à forte dose ( 5 Gy) en champ mixte, dans le cas où la composante neutron du champ de rayonnement est prépondérante $(\gamma / n=0,16)$, et dans le cas où elle est négligeable $(\gamma / n=11)$. 


\section{Dosimétrie en champ mixte et en milieu modérateur équivalent-tissu}

La mesure de la dose gamma en champ mixte neutron-gamma et en milieu hydrogéné pose de sérieuses difficultés en raison de la sensibilité de la plupart des dosimètres aux neutrons thermiques. Les dosimètres thermoluminescents tels que le $\mathrm{CaSO}_{4}$ et le $\mathrm{LiF}$ sont, de ce fait, pratiquement inutilisables à l'intérieur d'un mannequin équivalent-tissu, où l'abondance relative des neutrons thermiques est très importante (jusqu'à $80 \%$ de la fluence neutronique sous quelques centimètres de modérateur hydrogéné).

De ce fait, le signal délivré par le dosimètre peut être interprété comme résultant de la somme de deux termes, l'un correspondant à la dose gamma totale à mesurer, l'autre correspondant à la contribution parasite des neutrons thermiques et épithermiques. Dans ces conditions, la dose mesurée ( $D_{\text {mesurée }}$ ) peut être décomposée en deux termes:

$$
D_{\text {mesurée }}=h \cdot D_{\gamma \text { totale }}+k \cdot \Phi_{\mathrm{n}}
$$

Le coefficient $h$ associé à la dose $D_{\gamma \text { totale }}$ dépend de la réponse spectrale du dosimètre. On le rend égal à l'unité si le spectre de la composante photonique du champ ne comporte pas des énergies trop basses $(<100 \mathrm{keV})$ ou trop élevées $(>10 \mathrm{MeV}$ ) par rapport au rayonnement d'étalonnage, généralement le rayonnement gamma du ${ }^{60} \mathrm{Co}$.

Le facteur $k\left[\mathrm{~Gy} /\left(10^{12} \mathrm{n} / \mathrm{cm}^{2}\right)\right]$, correspondant au flux neutronique $\Phi_{\mathrm{n}}$, dépend de la nature du détecteur et de l'énergie des neutrons (Gibson, 1985): il exprime la sensibilité des matériaux thermoluminescents aux neutrons thermiques (tableau I) car la réponse parasite est essentiellement due à ces derniers.

\section{TABLEAU I}

Sensibilité aux neutrons thermiques de divers matériaux thermoluminescents.

Thermal neutron sensibility of various thermoluminescent materials.

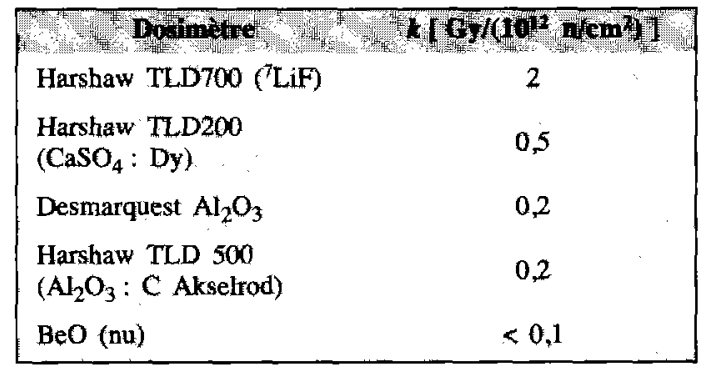


La sensibilité d'un dosimètre aux neutrons thermiques dépend de la composition isotopique des matériaux qu'il contient. Pour un même type de dosimètres elle peut varier d'un lot de fabrication à un autre en fonction de cette composition qui n'est pas absolument constante. C'est notamment le cas du fluorure de lithium, même appauvri en ${ }^{6} \mathrm{Li}$, en raison de la section efficace aux neutrons thermiques de cet isotope ( 950 barns). Même une teneur résiduelle garantie inférieure à $1 / 10000$ laisse subsister une sensibilité encore inacceptable. Le problème est le même avec les dosimètres dont le dopant est une terre rare telle que le dysprosium ou l'europium. Dans ce cas, on ne peut même plus intervenir sur la pureté puisque c'est le dopant lui-même qui est responsable de la sensibilité parasite. L'avantage de l'alumine thermoluminescente est qu'elle ne contient pas d'isotope dont la section efficace aux neutrons thermiques soit rédhibitoire pour les rayonnements les plus courants.

À titre d'exemple, comparons les réponses d'un dosimètre TLD700 ( $\left.{ }^{7} \mathrm{LiF}\right)$ et de la poudre d'alumine TL à l'intérieur d'un mannequin irradié par un champ mixte neutron-gamma de fission. Plaçons nous à $3 \mathrm{ou} 4 \mathrm{~cm}$ de profondeur dans le mannequin, dans l'axe de l'irradiation; c'est la région où la fluence des neutrons thermiques est maximale. Supposons que les doses neutrons rapide et gamma soient chacune égale à $1 \mathrm{~Gy}$. Le calcul montre que la fluence neutronique totale dans cette région est de $5 \times 10^{11} \mathrm{n} / \mathrm{cm}^{2}$ et que la part de neutrons thermiques peut atteindre $80 \%$ de cette fluence, soit $4 \times 10^{11}$ $\mathrm{n} / \mathrm{cm}^{2}$. Compte-tenu de leurs sensibilités respectives aux neutrons thermiques, le TLD700 indiquera une dose gamma de $(1+2 \times 0,4)=1,8 \mathrm{~Gy}$ c'est-à-dire près du double de la dose réelle, alors que l'alumine indiquera une dose maximum de $(1+0,2 \times 0,4)=1,08 \mathrm{~Gy}$, soit une erreur par excès inférieure à $8 \%$.

On pourrait penser, pour éliminer ce problème de réponse parasite, qu'il suffise de placer le dosimètre sous un écran absorbant les neutrons thermiques, c'est-à-dire contenant une forte proportion d'un isotope à forte section efficace de capture, par exemple du polyéthylène lithié $\left({ }^{6} \mathrm{Li}\right)$. Cette solution est pire que le mal car la dépression de fluence thermique créée dans le milieu par la présence, même localisée, de l'étui absorbeur s'étend bien au delà de ses limites physiques. Ceci se traduit par un déficit important de la dose gamma secondaire au niveau du dosimètre, les neutrons thermiques n'étant plus capturés par l'hydrogène du milieu ( $\sigma_{\text {capt }}=0,3$ barn), avec émission d'un photon de 2,2 $\mathrm{MeV}$, mais absorbés par le lithium sans réémission de photons. La seule solution est de choisir un dosimètre qui présente la sensibilité intrinsèque aux neutrons thermiques la plus faible possible; c'est le cas de $\mathrm{BeO}, \mathrm{Al}_{2} \mathrm{O}_{3}$, ou $\mathrm{Al}_{2} \mathrm{O}_{3}: \mathrm{C}$.

Étant donné que pour les basses énergies, la section efficace d'absorption photoélectrique est proportionnelle à $Z^{4} / E^{3}$, il conviendra en dosimétrie, afin de limiter la sur-réponse, de choisir un matériau dont le numéro atomique effectif $\left(Z_{\text {eff }}\right)$ soit aussi proche que possible de celui du milieu dans lequel il est placé (Attix et al., 1968). La courbe expérimentale de la figure 4 met en évidence ce type de sur-réponse dans le cas d'une plaque d'alumine dont 
le $Z_{\text {eff }}=10,2$ est supérieur à celui du tissu $\left(Z_{\text {eff }}=7,2\right)($ ICRU, publication 39). L'oxyde de béryllium dont la valeur du $Z_{\text {eff }}(7,13)$ est très voisine de celle du tissu, n'a pu être utilisé en raison de sa toxicité. Le choix de l'alumine s'appuie sur une réponse parasite moindre aux neutrons thermiques, une sensibilité suffisante au rayonnement gamma pour l'application envisagée et un coût relativement modique.

\section{Propriétés dosimétriques de l'alumine thermoluminescente}

Nous disposions de deux qualités de poudres d'alumine TL : l'une fournie par la société Desmarquest (Montreuil-sous-Bois, France) et l'autre fabriquée par M. Akselrod (Urals Polytechnical Institute, Russie), réputée très sensible au rayonnement photonique (Akselrod et al., 1990). Après une étude préliminaire, nous n'avons pas retenu l'alumine Akselrod car sa réponse en dose n'est linéaire que jusqu'à deux grays. D'autre part, nos expériences ont montré que lorsque la vitesse de chauffage passe de quelques kelvins par seconde à plus de $10000 \mathrm{~K} \mathrm{~s}^{-1}$ (chauffage laser), l'intégrale du signal de TL diminue d'un facteur de l'ordre de 50. Cette diminution a été attribuée à la baisse du rendement de luminescence associée à une augmentation de la température. Enfin, sa sensibilité aux UV ne facilite pas le procédé de fabrication des plaques ainsi que leur utilisation. Les résultats que nous présentons ont donc été obtenus avec la poudre Desmarquest.

L'intégrale du signal TL décroît significativement lorsque la taille des cristaux d'alumine devient inférieure à 40 micromètres (Fig. 1). La recherche d'un compromis entre sensibilité, homogénéité et reproductibilité des plaques dosimétriques nous a conduit à choisir une granulométrie de 50 micromètres.

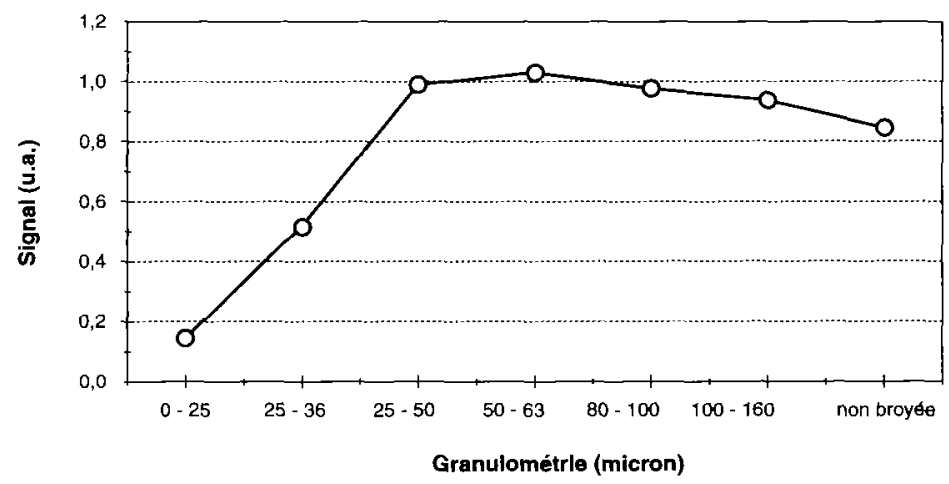

Fig. 1. - Intensité de la thermoluminescence de l'alumine Desmarquest en fonction de la granulométrie (irradiation au ${ }^{60} \mathrm{Co}$ ).

Thermoluminescent intensity of Desmarquest alumina as a function of granulometry $\left({ }^{60} \mathrm{Co}\right.$ exposure). 
L'étude expérimentale des plaques d'alumine que nous avons réalisées montre que l'intégrale du signal de TL est proportionnel à la dose (Fig. 2) ; le seuil de détection est de l'ordre de quelques dizaines de milligrays ; le phénomène de saturation du matériau apparaît au voisinage de $10^{3} \mathrm{~Gy}$. La décroissance de l'intégrale du signal de TL au cours du temps (fading) est de $15 \%$ en un mois, à température ambiante et dans l'obscurité (Fig. 3). L'alumine est connue pour sa sensibilité aux UV, caractérisée par deux comportements différents (Iacconi et al., 1986 ; Lapraz et al., 1988) :

- Un film non irradié soumis à un éclairement UV présente une prédose.

- Un film déjà irradié s'efface lorsqu'il est soumis à un flux d'ultraviolet.

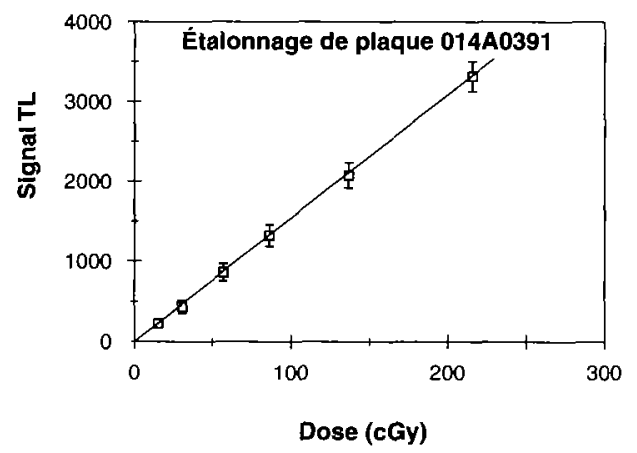

Fig. 2. - Réponse au chauffage laser de l'alumine thermoluminescente Desmarquest en fonction de la dose, pour une irradiation au ${ }^{60} \mathrm{Co}$.

Thermal heating response of Desmarquest thermoluminescent alumina as a function of doses, for ${ }^{60} \mathrm{Co}$ exposure.

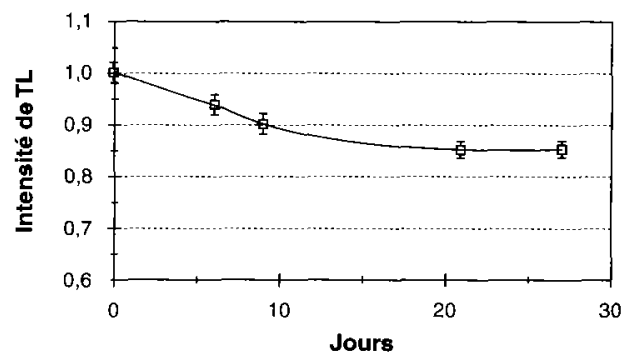

Fig. 3. - Décroissance au cours du temps du signal de $T L$ correspondant au pic $260{ }^{\circ} \mathrm{C}$ sur des dosimètres d'alumine (Desmarquest) irradiés au ${ }^{60} \mathrm{Co}$, lus en chauffage linéaire sans préchauffage (avec un stockage des dosimètres dans l'obscurité).

Time decreasing of $260^{\circ} \mathrm{C}$ TL peak of Desmarquest alumina for ${ }^{\circ 0} \mathrm{Co}$ exposure. Linear heating readout, without pre-heating and with stocking dosemeters in darkness. 
Dans le cas de l'alumine Desmarquet, ces phénomènes ne sont pas sensibles si le matériau n'est pas exposé à un flux intense ou de longue durée. Cependant les plaques devront être stockées à l'obscurité avant et après irradiation.

La reproductibilité entre deux fabrications de films et entre les films d'une même fabrication est actuellement au mieux de l'ordre de $5 \%$.

Ajoutons que la cartographie à l'aide de films minces permet de diminuer la sur-réponse à basse énergie due à la différence de numéro atomique effectif entre le dosimètre et le milieu équivalent-tissu constituant le mannequin (Fig. 4). En effet, l'épaisseur de la couche sensible du détecteur (50 micromètres) est du même ordre de grandeur que le parcours des électrons secondaires provenant du milieu dans lequel il est placé, et ceci jusqu'à de basses énergies (respectivement 125 et 38 micromètres pour des électrons secondaires de 100 et $50 \mathrm{keV}$ ). Contrairement à un dosimètre qui se présente sous forme de poudre ou de pastille, dans le cas des plaques les électrons secondaires ne perdent qu'une fraction de leur énergie en traversant la couche. On peut donc considérer, conformément à la théorie de Bragg-Gray (Attix et al., 1968), que la présence du film d'alumine TL ne perturbe pratiquement pas le milieu malgré la différence de $Z_{\text {eff }}$ avec le tissu $(10,2$ par rapport à 7,2$)$.

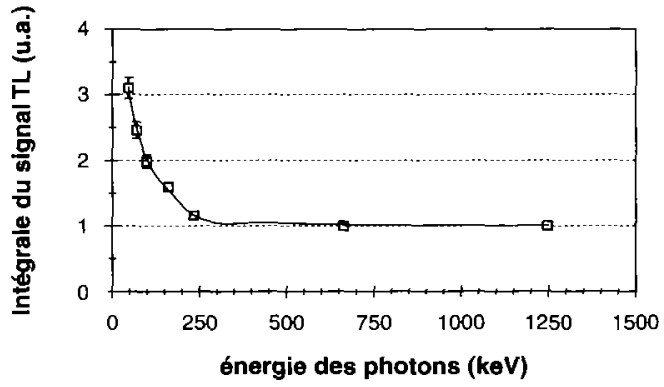

Fig. 4. - Réponse expérimentale en ênergìe de l'alumine Desmarquest déposée sur un film lue par chauffage laser, par rapport à l'équivalent-tissu, pour un même Kerma dans l'air (sources d'irradiation : $\mathrm{RX},{ }^{137} \mathrm{Cs},{ }^{60} \mathrm{Co}$ ).

Experimental energy response of $T L$ film Desmarquest alumina read by laser heating, respect to tissue-equivalent, for the same air kerma (irradiation sources: $\mathrm{X}$ rays, ${ }^{137} \mathrm{Cs}$, $\left.{ }^{60} \mathrm{Co}\right)$.

\section{Fabrication et lecture des films pour cartographie de dose}

Les dimensions des plaques TL que nous avons utilisées pour nos expériences sont comprises entre $5 \times 5 \mathrm{~cm}^{2}$ et $30 \times 30 \mathrm{~cm}^{2}$ (Servière, 1993). La poudre d'alumine monocristalline Desmarquest est déposée par sérigraphie sur une 
épaisseur de 50 micromètres, sur un support souple de kapton (polyimide) dont l'épaisseur est aussi de 50 micromètres. Ce support a été choisi en raison de sa bonne tenue en température, il a en plus l'avantage d'être un polymère ne comportant pas d'éléments à forte section efficace de capture pour les neutrons thermiques. Ce support est bien adapté à la dosimétrie gamma, en champ mixte neutron-gamma, dans des mannequins, car il ne perturbe ni le champ neutron, ni le rayonnement gamma.

La lecture s'effectue au moyen d'un laser $\mathrm{CO}_{2}$ de chauffage dont le faisceau ( $\lambda=10,6$ micromètres) est focalisé sur la plaque. Le diamètre du point d'impact dépend de l'objectif utilisé, il est compris entre 300 et 1000 micromètres. L'énergie fournie par le laser $\mathrm{CO}_{2}$ au point de focalisation du faisceau IR sur la plaque TLD provoque une élévation de la température de quelques centaines de degrés en quelques millisecondes.

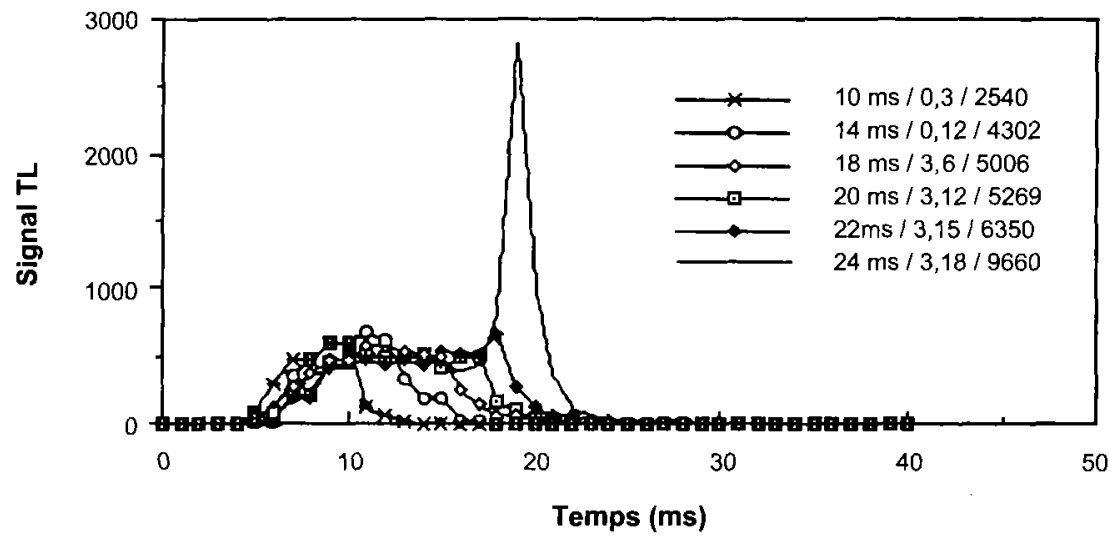

Fig. 5. - Courbes de thermoluminescence en chauffage laser obtenues pour différents temps de chauffe en différents points d'un même film d'alumine Desmarquest (irradiation aux $R X$ de $50 \mathrm{keV}$ ). Les valeurs reportées correspondent dans l'ordre, au temps de chauffage, aux coordonnées (en $\mathrm{mm}$ ) du point lu, et à la valeur de l'intégrale du signal de TL. Dans l'exemple présenté, lorsque le temps de chauffage excède $18 \mathrm{~ms}$, il apparâ̂t une contribution importante de l'émission corps noir qui peut conduire à la détérioration du dosimètre. Dans cet exemple un temps de chauffage inférieur ou égal à $15 \mathrm{~ms}$ devra être choisi.

Thermoluminescence glow curve in laser heating for various heating times (irradiation with $50 \mathrm{keV} \mathrm{X}$ rays). The reported values are heating time, coordinates $(\mathrm{mm})$ and value of integrated TL signal. In this example, for heating time above $18 \mathrm{~ms}$, the TL material begins to burn. The choice of an heating time should be lower than $15 \mathrm{~ms}$.

Le système de détection est constitué d'un photomultiplicateur, muni de filtre, qui convertit l'émission de thermoluminescence en courant. Ce signal numérisé permet de visualiser la courbe de TL. L'intégrale du signal est éta- 
lonnée en dose absorbée. Le film est déplacé pas-à-pas, ce qui permet d'obtenir la distribution, sur la surface de la plaque, de l'intégrale du signal de thermoluminescence, proportionnel à la dose absorbée.

Le principe du montage et son fonctionnement détaillé ont été présentés dans des publications antérieures (Bräunlich et al., 1981 ; Gasiot et al., 1982 ; Servière, 1993).

L'optimisation des conditions de lecture (puissance du laser et temps de chauffage) est déterminée expérimentalement à partir de la courbe de l'intensité de thermoluminescence en fonction du temps. Nous donnons sur la figure 5 les variations expérimentales du signal de thermoluminescence en fonction du temps pour diverses durées de chauffage. Ces temps de chauffage sont compris entre $14 \mathrm{~ms}$ et $18 \mathrm{~ms}$; au-delà de $22 \mathrm{~ms}$ il y a dégradation du dosimètre par combustion.

\section{Expériences et résultats}

Le problème que nous proposions de résoudre était d'effectuer des cartographies de dose dans les plans de coupe d'un mannequin anthropomorphe, de type «plastinaut » utilisé par la NASA pour les études des effets des rayonnements cosmiques sur les astronautes. Ce mannequin $(1,70 \mathrm{~m}, 70 \mathrm{~kg})$ est composé d'un polymère équivalent-tissu pour les neutrons et les photons et contient un squelette réel. La source de rayonnement est le réacteur expérimental SILENE de l'Institut de protection et de sûreté nucléaire (CEA/Valduc, France), utilisé pour les études d'accidents de criticité et de radiobiologie. Les doses absorbées délivrées dans l'air lors d'un tir sont comprises entre 0,1 et $10 \mathrm{~Gy}$.

Le réacteur peut être configuré de deux manières afin de favoriser l'une ou l'autre des deux composantes du rayonnement émis.

- Équipé d'un écran cylindrique extérieur en polyéthylène cadmié (SILENE/PE), la composante neutronique du champ de rayonnement est réduite par thermalisation et absorption des neutrons ; le rapport de la dose gamma sur la dose neutron à l'air libre à trois mètres est de $11(\gamma / n=11)$.

- Muni d'un écran de plomb (SILENE/Pb) atténuant la composante photonique du champ, le rapport gamma sur neutrons à l'air ljbre à trois mètres est de 0,16 . Dans cette configuration, un réflecteur en acier (guérite) peut être disposé derrière le mannequin afin de créer un champ plus omnidirectionnel simulant celui que l'on obtiendrait après propagation dans l'air à plusieurs centaines de mètres d'une source de fission de haute intensité.

Lors de l'irradiation, le film thermoluminescent est positionné entre deux tranches du mannequin au niveau de l'abdomen. Le mannequin est placé à 
3 mètres de la source. Une représentation schématique du positionnement du détecteur par rapport à la source est donnée en figure 6 .

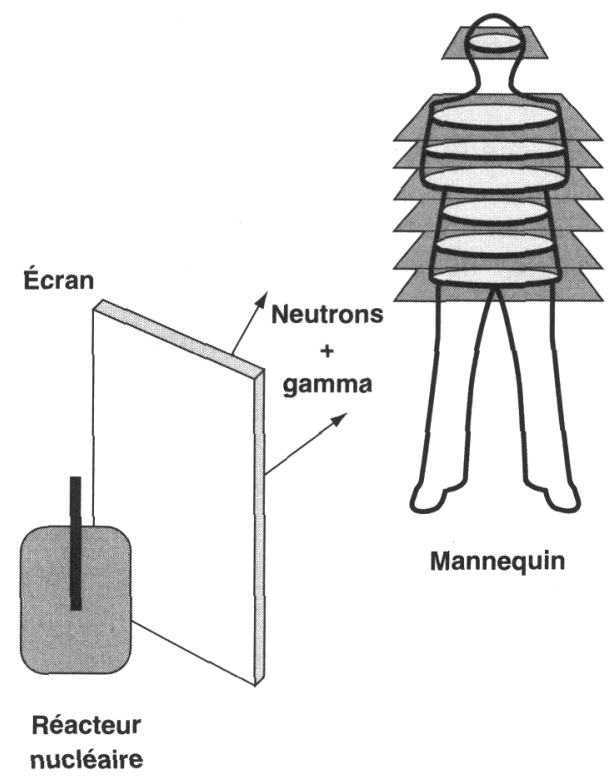

Fig. 6. - Géométrie de l'irradiation; positionnement des plaques dans le mannequin. Irradiation geometry; TL plates positioning in the phantom.

Une carte de dose dans une coupe horizontale au niveau de l'estomac (tranche $19 \mathrm{du}$ «plastinaut») est représentée sur la figure 7. Il s'agit de la configuration $\mathrm{SILENE} / \mathrm{Pb}$, avec un réflecteur en acier derrière le mannequin.

La figure 8 représente le profil de dose gamma totale suivant un axe antéropostérieur dans le mannequin. Les courbes présentées sont normalisées à la valeur de la dose au niveau de la face avant du mannequin. Cette représentation permet de comparer les variations de l'intégrale du signal de TL en fonction de la profondeur dans le mannequin, en s'affranchissant de la différence de réponse en dose de chaque plaque ainsi que de la dose totale délivrée pour chacune des irradiations.

Lors de l'irradiation en champ de rayonnement gamma (configuration SILENE/PE), on observe l'atténuation caractéristique de la dose dans un milieu atténuateur équivalent-tissu soumis à une irradiation gamma de type ${ }^{60} \mathrm{Co}$. Cette décroissance est due à l'action combinée de l'atténuation du rayonnement gamma par le milieu et de l'accroissement de la distance à la source. 


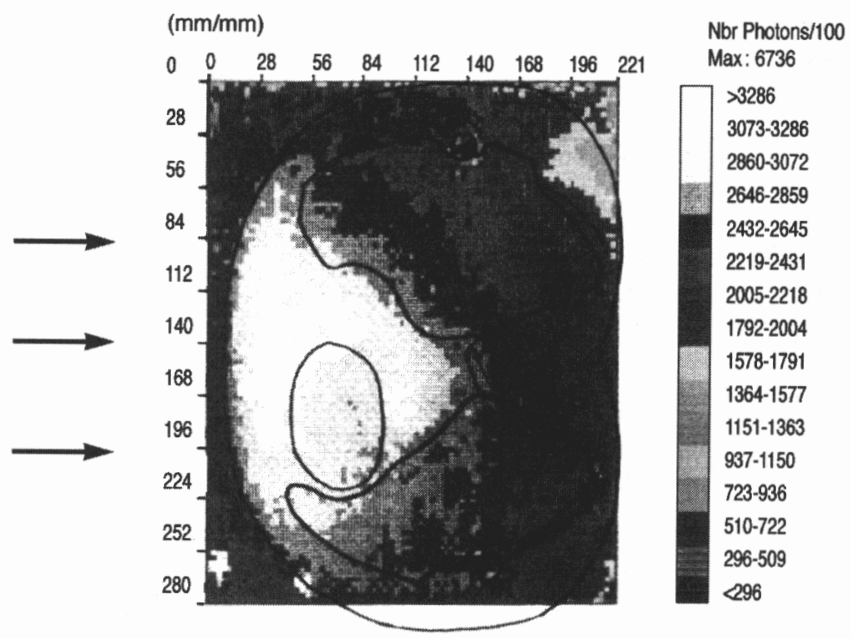

$\operatorname{Min}: 82$

Fig. 7. - Cartographie de la répartition de la dose gamma dans un plan de coupe abdominal d'un mannequin, disposé devant un réflecteur d'acier, soumis à une irradiation mixte neutron-gamma auprès du réacteur Silène avec écran de plomb.

Gamma dose mapping in an abdominal tranverse section of a phantom, set out in front of a steel reflector, irradiated in a mixed neutron-gamma field (Silene reactor, with lead shield).

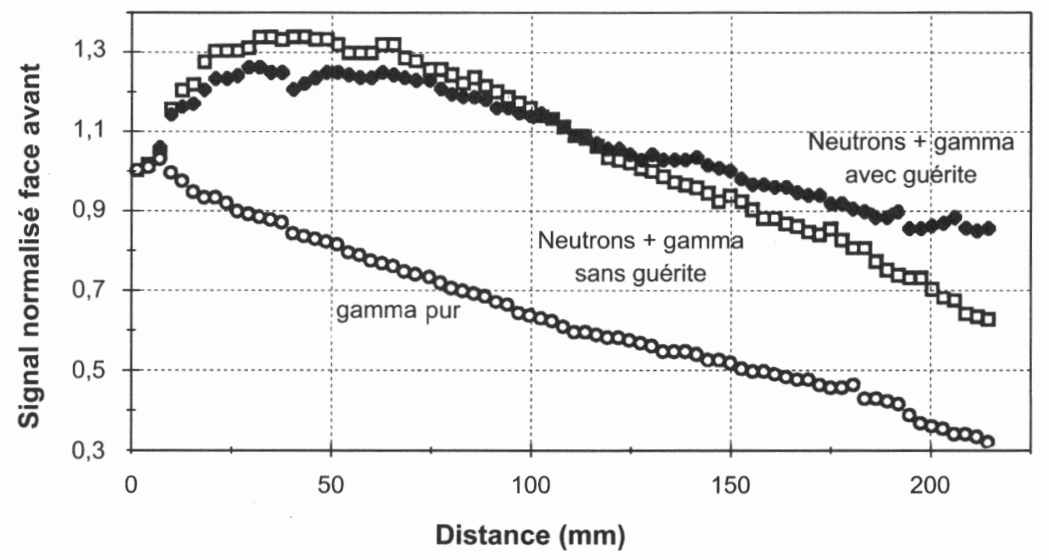

Fig. 8. - Répartition de dose obtenue dans chacun des mannequins suivant une coupe antéropostérieure dans l'axe du faisceau.

Dose distribution in each phantom on the radiation axis. 
En rayonnement mixte neutron + gamma (configuration SILENE/Pb), la décroissance de l'intégrale du signal de TL traduit la combinaison de trois composantes :

- la distribution de la dose due au rayonnement gamma issu de la source ;

- la distribution de la dose due à une source diffuse, à l'intérieur du mannequin, de rayonnement gamma secondaire, créée par les captures radiatives des neutrons thermiques par les noyaux d'hydrogène du milieu modérateur ;

- une sur-réponse du dosimètre due à la faible mais non nulle sensibilité de l'alumine aux neutrons thermiques. Cette sur-réponse est inférieure à $10 \%$ dans la zone du mannequin où la fluence des neutrons thermiques est maximale, c'està-dire à une profondeur d'environ $4 \mathrm{~cm}$.

La mise en place d'un réflecteur en acier disposé à l'arrière du mannequin permet d'augmenter la contribution du rayonnement rétrodiffusé, afin de se placer dans des conditions expérimentales plus réalistes. Ceci a pour effet d'augmenter la dose totale dans le mannequin et d'en diminuer le gradient de dose.

\section{Conclusion}

Ces résultats illustrent les possibilités de la cartographie de dose gamma en champ mixte neutron-gamma utilisant des plaques d'alumine. Elle permet de relever la topographie complète de la dose dans des coupes anatomiques traversant des milieux hétérogènes, même dans des champs mixtes en raison de la faible sensibilité de l'alumine aux neutrons thermiques $\left(2 \times 10^{-10} \mathrm{mGy} / n_{\mathrm{th}}\right)$ très abondants en milieu fortement modérateur.

Actuellement, la précision obtenue dans la détermination des doses à l'intérieur de mannequins avec ces films d'alumine TL, est du même ordre de grandeur que celle des dosimètres TL constitués de la même alumine lus par chauffage conventionnel. Cependant, ces derniers ne permettent pas d'obtenir une résolution spatiale aussi bonne que les films TL. Si la quantité de matière dosimétrique des capteurs TL frittés ou en poudre leur confère une meilleure sensibilité, cette quantité les pénalise du point de vue de la réponse spectrale.

Il convient de signaler que la qualité des mesures avec les films doit beaucoup au soin particulier apporté à la mise au point du couple film-lecteur laser. La cartographie de dose utilisant des plaques TL chauffées par laser, peut être adaptée pour répondre à de nombreux problèmes de dosimétrie lorsqu'un problème de localisation ou de résolution spatiale se pose. Il est cependant nécessaire de choisir le film TL le mieux adapté au champ de rayonnement et à l'application considérée. Ceci est rendu possible par la grande variété de matériaux TL disponibles et grâce à la possibilité de réaliser des films de dimensions quelconques. Un autre avantage de la cartographie par TL est la rapidité 
avec laquelle sont obtenues les mesures, typiquement 40 minutes pour une plaque $100 \times 100$ points.

Remerciements. Ce travail a été financièrement soutenu par la DRET. Nous tenons particulièrement à remercier M. J. Laugier et Mme A. Serbat du Centre d'Études du Bouchet (DGA, France) pour leur aide scientifique et technique, ainsi que M. P. Le Dorze, physicien d'hôpital, pour sa participation à ce travail.

\section{RÉFÉRENCES}

Akselrod M., Kortov V.S. et al. (1990) Highly sensitive TL anion-defective $\alpha$ - $\mathrm{Al}_{2} \mathrm{O}_{3}$ single cristal detectors. Radiation Protection Dosimetry, 32, 15-20.

Attix F., Roesch W., Tochilin E. (1968) Radiation dosimetry. Vol. I, II, III. (Academic press, $2^{\mathrm{e}}$ édition).

Bräunlich P., Gasiot J., Fillard J.P., Castagne M. (1981) Laser Heating of Thermoluminescent Dielectric Layers. Appl. Phys. Lett, 39 (9) 769, 771.

Cameron J. R., Suntharaglian N., Kenney G. N. (1968) Thermoluminescence Dosimetry, University of Wisconsin Press.

Gasiot J. , Bräunlich P., Fillard J. P. (1982) Laser Heating in Thermoluminescence Dosimetry. J. Appl. Phys., 53, 5200-5208.

Gibson J.A.B.(1985) The relative tissue-kerma sensitivity of thermoluminescent materials to neutrons. CEE publication.

Iacconi P., Lapraz D., Keller P., Portal G., Barthe J. (1986) Photo-induced thermoluminescence of Xirradiated $\alpha-\mathrm{Al}_{2} \mathrm{O}_{3}$ dosimetric properties. Radiation Protection Dosimetry, 17, 475-478.

ICRU (1985) Determination of dose equivalents resulting from external radiation sources. ICRU 39 publication.

Lapraz D., Iacconi P., Sayadi Y., Keller P., Barthe J., Portal G. (1988) Some thermoluminescence properties of an $\alpha-\mathrm{Al}_{2} \mathrm{O}_{3}$ sample. Sensitization effects. Physical Status Solidi (a), 108, 783-794.

Randall J. T., Wilkins M. H. F. (1945) The phosphorescence of various solids. Proc. R. Soc. London, A184, 347.

Servière H. (1993) Cartographie de dose gamma en champ mixte neutron-gamma en milieu modérateur : mise au point d'un capteur thermoluminescent et d'un lecteur laser, Université Montpellier II, Montpellier (France). 